\title{
Status foundations: Further consideration of the role of 'dominance' and the relative importance of cost infliction and benefit generation
}

\author{
Patrick Durkee*a, Aaron Lukaszewski ${ }^{\mathrm{b}}$, and David Buss ${ }^{\mathrm{a}}$

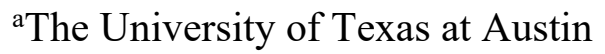 \\ ${ }^{\mathrm{b}}$ California State University, Fullerton
}

This preprint is a reply to "Dominance is necessary to explain human status hierarchies - Extended online version and supplemental" by Cheng et al. (2021), available at: psyarxiv.com/w28nt/

*Address correspondence to Patrick Durkee (pdurkee@utexas.edu) 


\section{Introduction}

We recently published an empirical article examining the psychological foundations of human status allocation (Durkee et al., 2020). In the study, people made inferences about the extent to which 240 status-affecting items (acts, traits, or events) indicate an individual's ability or willingness to (a) generate benefits for others or (b) inflict costs on others. In order to test the competing predictions from theories of human status acquisition, we examined the unique predictive impacts of benefit generation inferences and cost infliction inferences, respectively, on the extent to which those same 240 items were rated as being likely to increase or decrease a person's status within 14 diverse nations around the world (Buss et al., 2020). The results demonstrated that, when benefit generation inferences and cost infliction inferences were competing predictors, benefit generation inferences accounted for a large majority of the variance in status allocations across nations over and above cost infliction, whereas cost infliction inferences were weak unique predictors over and above benefit generation. From these results, we concluded that benefit generation inferences constitute the main psychological foundation of human status allocations, and that associations between cost infliction inferences and status are primarily due to overlap with benefit generation inferences.

In their commentary on our article, Cheng et al. $\left(2021^{\mathrm{a}}\right)$ present two reasons to doubt our conclusion that benefit generation is the main psychological foundation of status allocation in human groups, which they elaborate upon in an extended supplemental commentary (Cheng et al., 2021 ${ }^{\mathrm{b}}$ ). First, Cheng and colleagues (hereafter, CTH) claim that collinearity among our predictors prevent us from examining the relative roles of being perceived as willing or able to inflict costs on or generate benefits for others. Second, CTH summarize previous research that they interpret as evidence that cost infliction and benefit generation are similarly important for attaining status, which they argue are inconsistent with our findings.

In this paper, we provide an extended reply to CTH that counters these two points. Part 1 provides extended discussion of the shortcomings of "dominance" as operationalized in extant literature - which in our view severely limits the conclusions that can be drawn about the impacts of costs infliction on status - and sketch a conceptual framework for refining our understanding of whether and how cost infliction begets status. Part 2 addresses concerns about the impact of collinearity on our conclusion by presenting results from several additional analyses that address collinearity among our predictors. These new analyses provide further support for our original conclusion - that benefit generation is a more important psychological foundation of human status allocations than cost infliction. 


\section{$\underline{\text { Part } 1}$}

\section{Refining Dominance: On the need to distinguish pure cost infliction from benefit generation via cost infliction}

CTH highlight several previous empirical investigations of the association of "dominance" and "prestige" with status, which they claim support a similarly large role of cost infliction and benefit generation in obtaining status. We do not necessarily disagree with CTH's title thesis that "dominance is necessary to explain human status hierarchies". But there is a large disconnect between the theoretical concept of dominance (i.e., status rooted in threatened or actualized cost infliction) and common operationalizations of "dominance" in empirical studies, which broadly reflect a heterogeneous mix of assertive, selfish, controlling, coercive, aggressive, and even complaisant tendencies (Ketterman \& Manor, 2021). As we argue below, because these operational definitions rely on underspecified folk conceptions of "dominance", it is challenging to use such measures to make clean inferences about the role(s) of cost infliction in status attainment. In our view, there are two non-mutually exclusive ways in which dominance could be conceptually and operationally partitioned to better understand the role of cost infliction in status attainment:

(1) Pure Cost Infliction (PCI): Individuals acquire and maintain status by eliciting fear in group members about the costs he or she might inflict on them if they do not defer.

(2) Benefit Generation via Cost Infliction (BGCI): Individuals acquire and maintain status due to their perceived ability and willingness to selectively inflict costs in (mostly) in-group-beneficial ways, such as punishing free-riders or harming rival out-group members.

From the standpoint of the dominance-prestige model, it is unclear whether BGCI should be regarded as dominance or prestige. As noted in Henrich and Gil-White's (2001) seminal work distinguishing dominance and prestige, "prestige-deference evoked by one's great skill or knowledge in inflicting costs (e.g., violence) should not be confused with the dominancedeference created by the fear that one may become a victim of that violence." (p.172). Thus, within the confines of the dual-pathway dichotomy, we suggest that status derived from BGCI could be considered prestige, since the mediating factor between cost infliction and status is net benefit generation (i.e., prestige). This would be consequential to the extent that it could be established that BGCI accounts for most instances in which cost infliction results in lasting status within a community. As it stands in the dominance-prestige literature, (i) most characterizations of status derived from dominance appear to simply assume that it occurs primarily through PCI, and (ii) aspects of PCI and BGCI are conceptually and operationally lumped together as "dominance". Regardless of whether BGCI is considered "dominance" or a sub-type of "prestige", we contend that distinguishing between BGCI and PCI is ontologically and functionally important. Indeed, it was the prospect of helping to accomplish this objective that partly motivated our recent study regarding the psychological foundations of human status allocation (Durkee et al., 2020) — which is to our knowledge the only study that has attempted to distinguish different forms of cost infliction and benefit generation. 


\section{Does extant research support a similarly large role of cost infliction and benefit generation in status attainment?}

Having considered these conceptual issues, let us return to CTH's claim that the extant literature supports a dominance-prestige model in which cost infliction and benefit generation are independently viable pathways to community-wide status. While previous studies cited by CTH as evidence for the dominance-prestige model (e.g., Anderson et al., 2020; Brand \& Mesoudi, 2019; Cheng et al., 2013; Garfield \& Hagen, 2019; Redhead et al., 2019; von Rueden et al., 2008, 2014) are laudable in many respects, none of them cleanly separated PCI and BGCI (nor were they necessarily designed to). Consequently, the extant evidence for "similarly large impacts" (Cheng et al., 2021 a, p.2) of benefit generation and cost infliction without benefit generation in status attainment across contexts and groups is weaker than it may appear.

Many of the studies cited as evidence for the role of cost infliction in status attainment rely on the dominance-prestige scale (Cheng et al., 2010). This scale includes items that, on their face, measure a mix of forceful (e.g., "He/she does NOT have a forceful or dominant personality"), controlling (e.g., He/she enjoys having control over other members of the group"), assertive (e.g., "He/she often tries to get his/her own way regardless of what others in the group may want"), and aggressive (e.g., "He/she is willing to use aggressive tactics to get his/her way") tendencies, as well as the elicitation of fear in others ("Members of your group are afraid of him/her"). As we have argued above, these items can reflect both PCI and BGCI strategies. Thus, even though both components of the dominance-prestige scale are often associated with status, it is not clear whether dominance-status associations are driven by PCI, BGCI, or some combination of the two in these studies (e.g., Brand \& Mesoudi, 2019; Cheng et al., 2013; Redhead et al., 2019).

In considering the content of the dominance-prestige scale, it is important to note that individuals whose status is grounded in BGCI may be regarded with some ambivalence, given their combination of legitimate authority and aggressive formidability. For example, even if community members acknowledge the beneficial role played by a formidable leader who enforces local policies and administers punishments, they may still fear the prospect of being on the receiving end of such enforcement. As such, individuals whose status derives from BGCI may be described at the folk level as "dominant", "aggressive", "controlling"—and even perceived as unlikeable relative to someone who generates benefits without inflicting any costs. A corollary of this is that BGCI-based status may not necessarily be associated with the folk descriptions that are used to operationalize prestige (e.g., "others seek my advice on a variety of matters", "members of my group respect and admire me") by the dominance-prestige scale.

Other studies that CTH cite to support the dominance pathway, which do not rely on the dominance-prestige scale, are similarly ambiguous with respect to whether PCI or BGCI drive associations between dominance and status attainment. For instance, Anderson et al.'s (2020) longitudinal study measured dominant behaviors with items that do not specify to whom aggressive, assertive behaviors are directed (e.g., "not afraid to engage in conflict with others", willing to bully others to achieve important goals", "intimidates others"), which leaves open the possibility that these ostensibly "dominant" individuals obtain power not through PCI, but through BGCI — regardless of whether group members 'like' them for this or not. Although 
Garfield \& Hagen (2020) found that leadership among the Chabu was associated with being feared and perceived as intimidating by peers ${ }^{1}$, it is not clear that these perceptions are the source of their leadership position - being intimidating and feared may well be an outcome of the leaders' enhanced power to control group outcomes or greater coalitional support (von Rueden et al., 2019). While physical size is associated with leadership and community influence among the Tsimane (von Rueden, 2008, 2014), this says nothing about whether physically dominant men are attaining status from PCI, BCGI, or other forms of benefit generation (e.g., greater productivity) - in fact, experimental research suggests that the link between physical dominance and status is mediated by BGCI (Lukaszewski et al., 2016). In sum, previous research does not clearly demonstrate that status can be attained and maintained through PCI, let alone that cost infliction and benefit generation play similarly important roles.

The consequences of conflating PCI with BGCI are also apparent in the recent literature on dominance and prestige as distinct styles of leadership. Researchers have been puzzled by the apparent rise of "dominant" leaders across the globe (e.g., Kakkar \& Sivanathan, 2017). We propose that the preference of voters for such a leader is only puzzling if one assumes that the leader's status derives from the PCI pathway. But it clearly does not - it simply doesn't logically follow that individuals voted for putatively "dominant" leaders, such as Donald Trump, because they personally feared the prospect that he would inflict costs on them if they did not support him. We contend that individuals perceive "dominant" leaders as being able and willing to generate benefits for themselves and their coalition (e.g., job prospects, economic benefits, excluding potential competitors such as immigrants from sharing or partaking in in-group benefits). Indeed, even perceptions of leaders as "dominant" or "prestigious" are largely dependent on whether the perceptions are those of ingroup or outgroup members: According to a recent study by Jimenez et al. (2021), "people view political leaders who share their ideology as prestigious, and those who oppose their ideology as dominant, regardless of whether their ideology is liberal or conservative" (p.1).

Of course, our own study (Durkee et al., 2020) shares many limitations of previous research (e.g., cross-sectional self-report data), and it is possible that our status prompt does not capture all aspects of human status. Additional evidence will be required to determine whether we are correct about the utility of distinguishing between PCI and BGCI. But investigation of this distinction may help to better understand the relative importance of cost infliction and benefit generation in attaining status within the diverse range of human groups around the world. Research is needed that differentiates between PCI and BGCI and tests their relative importance alongside more general forms of benefit generation for determining status within human groups — in all their diverse forms.

\footnotetext{
${ }^{1}$ In contrast to Cheng et al.'s (2021) claim that this study supports a similarly large role of dominance and prestige, Garfield and Hagen (2020) also found that prestige was more strongly associated with leadership than was dominance, especially in Bayesian models that used weakly-informative priors instead of priors informed by studies of dominance and prestige in lab-based groups. Moreover, in the Chabu, dominance was not associated with leadership in women, which parallels our finding that cost infliction is not associated with women's status after controlling for its overlap with benefit generation.
} 


\section{Potential exceptions to the unimportance of pure cost infliction in status attainment}

As discussed above, we are skeptical of the prevailing notion that most putative observations of "dominance" in the extant literature reflect the PCI pathway. But we do not discount the possibility that PCI-based forms of status occur at some levels within in human groups. Below we highlight several exceptions to the relative unimportance of PCI.

Small and short-term groups. PCI could be used to gain and maintain influence and control within dyads or within small groups (e.g., Cheng et al., 2013) — especially if only a short-term group - wherein the collective formidability and motivation of the victims of threatened or actualized cost infliction is not sufficient to sanction PCI. However, as n-person groups become larger and more enduring, the collective leveling power and motivation of victims of repeated cost infliction radically diminishes the relative formidability of an individual deploying a status acquisition strategy grounded substantially in PCI (Boehm, 1993; Cheng, 2020; Gintis et al., 2015; Wrangham, 2021). Thus, we agree with the emerging evidence that PCI may be effective on its own in the short term in some delimited contexts, but it is unlikely to result in group-wide status that persists over time (Redhead et al., 2019; Cheng, 2020).

Competition for group-based status. A likely exception to the relative unimportance of pure cost infliction (PCI) in status acquisition exists at the level of competing subgroups within a larger population (e.g., ethnic groups, military bands, informal coalitions, cliques) in which the collective formidability of one group is greater than the collective formidability of another- and thus, collective PCI by the more formidable group cannot be effectively prevented by other groups. Similarly, a relatively small group may be able to maintain status via PCI over a much larger group if institutional factors are present that limit the ability of the larger group to solve the collective action problem of leveling the aggressively dominant coalition (e.g., if communication of shared interests is thwarted or emerging leaders are imprisoned or killed). In such instances, we expect that status at the population-level would appear to be derived from PCI, but status within each subgroup would still depend on the ability to generate benefits for their own coalition - whether via cost infliction or direct provisioning of benefits. In sum, PCIbased status pursuit likely requires allies. Correctly specifying the level of social organization at which costs are being inflicted or benefits are being generated is a crucial aspect of determining the extent to which PCI is an effective status attainment strategy within human groups.

Shamanism. Another interesting exception to the small role for PCI in acquiring and maintaining status in larger communities is apparent in the status of shamans. In small-scale societies, shamans frequently inflict costs on others in the community, including via physical aggression (Hess et al., 2010), that do not result in decreased status (Garfield et al., 2020). Recent work on the origins of shamanic beliefs suggests that belief in the magical abilities of certain individuals (shamans, witches) can evolve culturally, especially when some individuals benefit from those beliefs, and even when those beliefs are costly for most people who hold them (Singh, 2018). But shamanic status grounded partly in self-interested aggression may be an exception that proves the rule: if community-wide status is to be attained via PCI, the grammar of status allocation must be systematically circumvented (in this case, via the culturally-evolved institution of shamanism). Moreover, shamans may be perceived by group members as being able to generate many potential benefits, such as healing magic or protection from supernatural 
forces - regardless of whether these are in fact benefits that they can deliver. These perceptions of potential benefit generation ability may outweigh the perceived costs inflicted by shamans, and thus shamans may obtain and maintain their status despite their heightened propensity for PCI.

Residual correlations of cost infliction with status attainment. We also note that manifest associations between attained status and PCI may reflect an outcome of status attainment resulting from benefit generation, rather than a cause of status attainment itself. Status grounded in net benefit generation can be expected to afford many prerogatives (Henrich \& Gil-White, 2001). For instance, status can lead to increased tolerance of transgressions and privilege to demand preferential treatment from subordinates or followers (Kakkar et al., 2020; Polman et al., 2013). A residual association of cost infliction with status allocation might therefore exist because higher status affords more opportunity to inflict costs or withhold benefits from others in a self-serving manner, rather than because self-interested cost infliction per se resulted in higher status (Maner \& Mead, 2010). As an extreme example, an individual who is perceived to be the only community member capable of generating a specific valuable resource (e.g., protection from enemies) or modeling a specific valued skill (e.g., shamanic rituals), may be able to get away with demanding greater preferential treatment than they otherwise would if there were more individuals capable of generating that specific benefit. A residual association between cost infliction and status could also arise due to common causes between benefit-generating traits and aggressive behavior. For example, physically strong men may be more aggressive on average (e.g., Sell et al., 2009; Sell et al., 2016) and also have greater ability to produce benefits (e.g., Garfield and Hagen, 2020; Lukaszewski, 2013; von Rueden, 2014), so their status attainment could be residually correlated with aggressive tendencies even though benefit generation is driving the status attainment. In sum, the ratio of tolerated costs to expected benefits should still be heavily biased toward benefits for high status individuals - that is, high status individuals should on average be perceived as net benefit generators by those who defer to them.

\section{Conclusions}

The dominance-prestige model (Cheng et al., 2010; Cheng et al., 2013; Henrich \& GilWhite, 2001) represents a major conceptual advance for conceptualizing human status hierarchies. However, as we have argued, ambiguity in extant theory and measurement tools have prevented a full understanding of the unique and overlapping roles played by cost infliction and benefit generation in status allocation. Although extant studies finding an association between 'dominance' and status are consistent with the possibility that PCI is a viable strategy for attaining status, none demonstrate that the role of 'dominance' in status hierarchies is uniquely driven by pure cost infliction rather than by benefit generation through cost infliction, which is important because the theoretical concept of dominance is rooted in pure cost infliction. Our study (Durkee et al., 2020) attempted to partition the variance explained by cost infliction and benefit generation in status allocation, and - although our study is imperfect and represents only one source of evidence-it suggests that benefit generation is the primary foundation of human status. Additional research that distinguishes between PCI and BGCI will be necessary to probe the generalizability of this conclusion. 


\section{Part 2}

\section{Additional analyses addressing collinearity among our predictors of status allocations}

In light of CTH's concerns about collinearity among our predictors, we decided to explore additional statistical methods that can address collinearity to clarify the relative importance of cost infliction and benefit generation inferences in status allocations. Many methods have been developed to examine the relative importance of correlated predictors. Here, we apply four of them - dominance analysis, commonality analysis, ridge regression, and random forest modelsto our 14-nation status criteria and affordance perception data (for full descriptions of these data, see Buss et al., 2020; Durkee et al., 2019; Durkee et al., 2020). This multi-method analytic approach allows us to examine the relative importance of benefit generation and cost infliction inferences in explaining the impacts that personal characteristics are perceived to have on status in communities around the world while addressing collinearity in different ways. Across all four of these methods, the results are consistent with our original conclusion that benefit generation is a more important predictor of the perceived impacts of different personal characteristics on men's and women's status in samples from 14 nations than cost infliction.

\section{Dominance Analysis}

Dominance analysis was developed to identify which predictor in a set of predictors explains the greatest proportion of variance in a focal criterion (Budescu, 1993; Azen \& Budescu, 2003). Dominance analysis addresses collinearity by conducting an exhaustive set of regressions using different, non-redundant combinatorial sets of predictors, where the largest predictor-set contains the number of predictors minus one. For each of these submodels, the predictors that are of interest in the dominance analysis will each be entered independently to any model in which they are not already present, and the additional variance explained in the criterion above and beyond the variance accounted for by the combination of other predictors in the model is recorded. This additional unique contribution to the variance explained is compared in a pairwise manner to the unique contribution of each of the other predictors that are the focus of the dominance analysis across the exhaustive set of submodels.

Take our four original predictors - benefit generation ability (BGA), benefit generation willingness (BGW), cost infliction ability (CIA), and cost infliction willingness (CIW) - as a concrete illustration. The set of all possible models will contain an intercept only model, a subset of four models that each contain only one of the predictors (i.e., BGA, BGW, CIA, CIW), a subset of six models that will contain all non-redundant combinations of two predictors (i.e., BGA+BGW, CIA+CIW, BGA+CIA, BGW+CIW, $\mathrm{BGA}+\mathrm{CIW}, \mathrm{BGW}+\mathrm{CIA}$ ), and a subset of four models containing all non-redundant combinations of three predictors (i.e., $\mathrm{BGA}+\mathrm{BGW}+\mathrm{CIA}, \mathrm{BGA}+\mathrm{BGW}+\mathrm{CIW}, \mathrm{BGA}+\mathrm{CIA}+\mathrm{CIW}, \mathrm{BGW}+\mathrm{CIA}+\mathrm{CIW}$ ). To illustrate the pairwise comparison process, when the relative dominance of BGA and CIA is the comparison of interest, the additional contribution of BGA is compared to the additional contribution of CIA across four model subsets: (1) the null model; (2) the three models with only one predictor in which each of the two focal predictors is not already present (e.g., the contribution of CIA to models including only BGA, only $\mathrm{BGW}$, or only CIW); (3) the three models with two predictors in which each focal predictor is not present (e.g., the contribution of CIA to the BGA+BGW, BGA+CIW, and BGW+CIW models); and (4) the single model with three predictors in which the focal predictor is not present (e.g., the contribution of CIA to a the $\mathrm{BGA}+\mathrm{BGW}+\mathrm{CIW}$ model). This process of comparing the additional contribution of a pair of predictors across the complete set of submodels is repeated for each $i-j$ pair of predictors (BGA-BGW, CIA-CIW, BGA-CIA, BGW-CIW, BGA-CIW, BGW-CIA) to establish the dominance relationship between each pair of predictors. 
The dominance of one predictor over another is determined by the extent to which it contributes greater additional variance to comparable models (i.e., models with the same number of existing predictors) than its contender. Three levels of dominance can be established: complete dominance, conditional dominance, and general dominance. A predictor completely dominates another if its additional contribution to the criterion is greater than that contributed by its competitor in every subset model. A predictor is conditionally dominant if it accounts for greater additional variance than its competitor at any submodel level (e.g., all submodels with only three predictors), meaning that its dominance depends on the set of other predictors in the model. General dominance is established if the average additional contribution to variance explained across all sets of submodels for a predictor is greater than its competitor. The three dominance levels are hierarchical, such that complete dominance establishes conditional and general dominance, whereas conditional dominance establishes only general dominance, and general dominance is the lowest form of dominance.

To illustrate the three levels of dominance with our predictors, consider the BGA-CIA comparison. If BGA explains more additional variance in status allocations than does CIA in every submodel, BGA will have complete dominance over CIA. If the additional contribution of BGA to explaining status allocations is greater than that of CIA across some but not all submodels, then BGA establishes conditional dominance over CIA; here, the set of submodels in which BGA explains greater additional variance can yield insight into the conditions under which BGA is a more useful predictor than CIA. Finally, if the additional contribution of BGA to status allocation averaged across all model subsets is greater than the contribution of CIA averaged across submodels, then BGA establishes general dominance over CIA.

As reported in our formal published reply (Durkee et al., 2021), we used dominance analysis to assess the relative importance of our four original predictors as well as the composite variables used by CTH (i.e., composite of BGA and BGW, and composite of CIA and CIW). We conducted dominance analysis on our original data, separately for men's and women's status in each of the 14 nations in our sample. In order to obtain results that generalize better to new samples of status-affecting personal characteristics, we repeated each of the sex and country-specific dominance analyses in 2000 bootstrapped replicates and examined the proportion of replicates in which the different levels of dominance were established between predictors. We used the R package dominanceanalysis to conduct these analyses (Navarrete \& Soares, 2020).

Figures 1 and 2 show the proportion of 2000 bootstrapped samples in which each level of dominance was established across pairwise comparisons of both our individual predictors and the composite variables in explaining men's and women's status, respectively, in each of the 14 nations in our sample. The results show that the benefit generation composite used by CTH in their re-analyses completely dominates the cost infliction composite across nations in $100 \%$ of replicates for both men's and women's status, so conditional and general dominance of benefit generation is a given. 


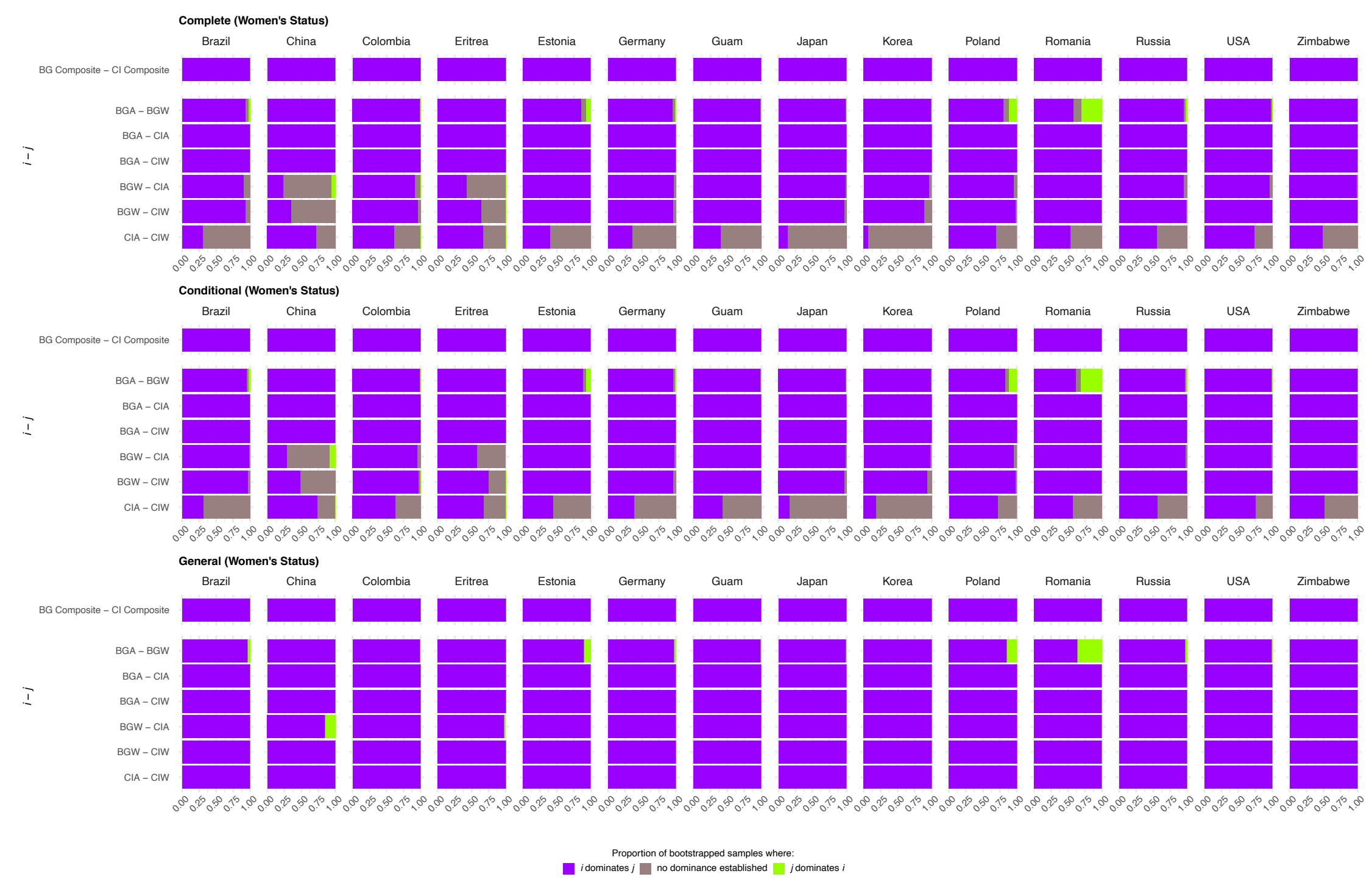

Figure 1. Results of dominance analyses showing the proportion of 2000 bootstrapped replicates in which each level of dominance was established between pairwise $(i-j)$ comparisons of predictors of the impacts of personal characteristics on women's status across nations. 


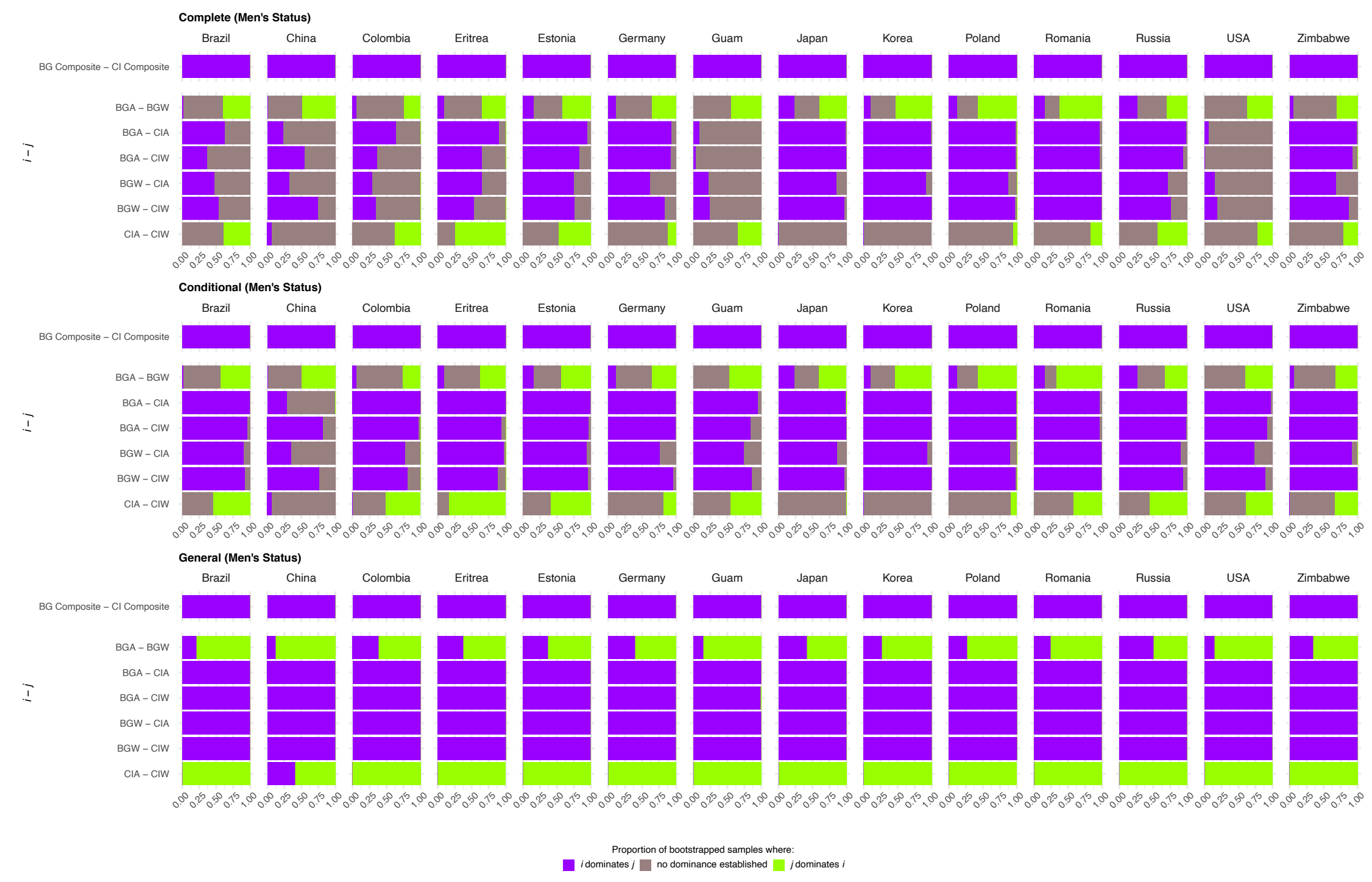

Figure 2. Results of dominance analyses showing the proportion of 2000 bootstrapped replicates in which each level of dominance was established between pairwise $(i-j)$ comparisons of predictors of the impacts of personal characteristics on men's status across nations. 
For individual predictors, the complete dominance of both BGA and BGW over CIA and CIW is reliably established in the vast majority of replicates for women's status across nations, with the exception of China and Eritrea where complete dominance could often not be established, and CIA completely dominated BGW in small percentage of replicates. Complete dominance of BGA and BGW over CIA and CIW was established in only about half of replicates for men's status and was highly variable across nations; however, in no replicates did CIA or CIW establish complete dominance over BGA or BGW. Conditional dominance of both benefit generation variables over the cost infliction variables is much more reliably established across replicates and countries for both men's and women's status; moreover, the sets of models in which dominance of either benefit generation variable over either of the cost infliction variables could not be established, tend to be in models that already include the other benefit generation variable as a predictor. Finally, the general dominance of both BGA and BGW over the CIA and CIW is established in 100\% replicates for men's status in every nation, and for women's status in all replicates across most nations except China and Eritrea. Taken together, the results of these analyses suggest that benefit generation is more useful than cost infliction for explaining the status impacts of personal characteristics on men's and women's status in communities around the world, with some potential variation across cultures that could be explored with future research.

\section{Commonality Analysis}

Commonality analysis addresses collinearity by decomposing the percentage of total variance accounted for by predictors into common and unique variance components for all combinations of predictors (Jernstedt, 1980; Ray-Mukherjee et al., 2014). The common variance components provide an understanding of the percentage of total variance accounted for in the criterion that can be attributed to shared information among different sets of predictors. The unique variance components show what proportion of total variance accounted for can be attributed to the information a predictor contains that is not shared by other predictors.

We re-examined the common and unique variance in status allocations accounted for by cost infliction and benefit generation using commonality analysis. To better generalize the results to new samples of status-affecting characteristics and generate confidence intervals for the estimates of different variance components, we conducted the commonality analyses in 2000 bootstrapped replicates separately for men and women in each nation. We used the commonalityCoefficients function in the R package yhat to conduct our analyses (Nimon et al., 2008).

Figure 3 shows the proportion of unique and common variance accounted for in the impacts of personal characteristics on men's and women's status within each nation using different sets of our original predictors, as well as the composite variables used by CTH. The results show that most of the variance in status allocations around the world that is accounted for by inferences of benefit generation and cost infliction affordances can be attributed to common variance; that is, information about cost infliction ability also tends to provide information about benefit generation ability and vice-versa. However, inferences of benefit generation account for a considerable share of unique variance in status allocations, while the unique information provided by cost infliction affordances - information unassociated with benefit generation - accounts for relatively little in status allocations. This is consistent with our original conclusion that much of the association between cost infliction and status allocation can be attributed to overlapping variance with benefit generation. 

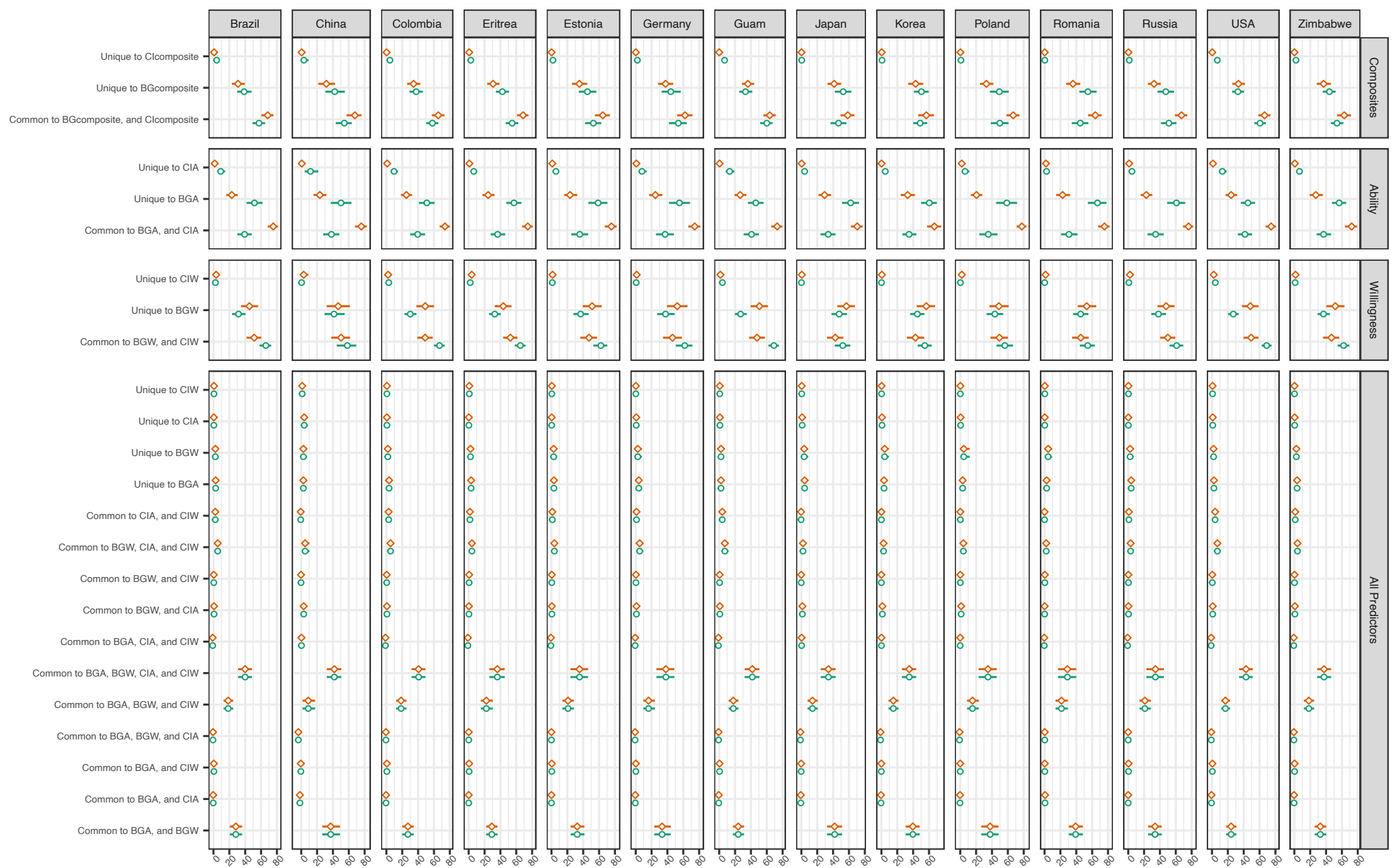

Percentage of total variance accounted for

\& Men's Status \& Women's Status

Figure 3. The results of commonality analysis across 2000 bootstrapped replicates, showing the average variance (and $95 \%$ CI) accounted for in men's and women's status across 14 nations that can be attributed to common variance among combinations of predictors and unique variance of single predictors. 


\section{Ridge Regression}

Ridge regression provides more stable estimates of coefficients than OLS regression by reducing the manifest correlation among predictors and suppressing the collinearity (Ullah et al., 2018). Ridge regression is a biased alternative to the least squares estimator for regression coefficients (Hoerl \& Kennard, 1970). It is a biased method in that, in the case of highly correlated predictors, the coefficients of weaker predictors will be pushed away from zero, while the coefficients of stronger predictors are pull downwards towards zero (Vanhove, 2021). The degree of shrinkage is determined by a biasing penalty $(k)$. For every regression solution, there is an optimal value of $k$ that results in lowest mean square error of the estimates. Generalized cross-validation is commonly used to identify the optimal setting of the biasing penalty (Golub et al., 1979; Ullah et al., 2018).

We used ridge regression to further explore the associations of cost infliction and benefit generation to status allocations. We ran separate ridge regression models to predict men's and women's status in each nation. To identify the optimal biasing penalty, we conducted a wide parameter sweep for each model with values of $k$ ranging from 0 to 50 in .01 increments and chose the value that had the lowest mean square error according to the generalized cross-validation method. Ridge regression does not provide standard errors or $\mathrm{p}$-values for the coefficient estimates, so we conducted 2000 bootstrapped replicates of each model to generate $95 \%$ confidence intervals for each coefficient estimate. We used the lm.ridge function in the MASS package to conduct the ridge regressions (Ripley et al., 2013).

Figure 4 shows the mean coefficient estimate and 95\% bootstrapped confidence intervals for each predictor of men's and women's status in each country across different combinations of predictors. In line with the re-analyses conducted by $\mathrm{CTH}$, cost infliction variables tended to have a non-zero association with men's and women's status across nations in models with just one benefit generation and one cost infliction variable; however, this estimate for cost infliction remains substantially smaller than benefit generation in all model comparisons. 


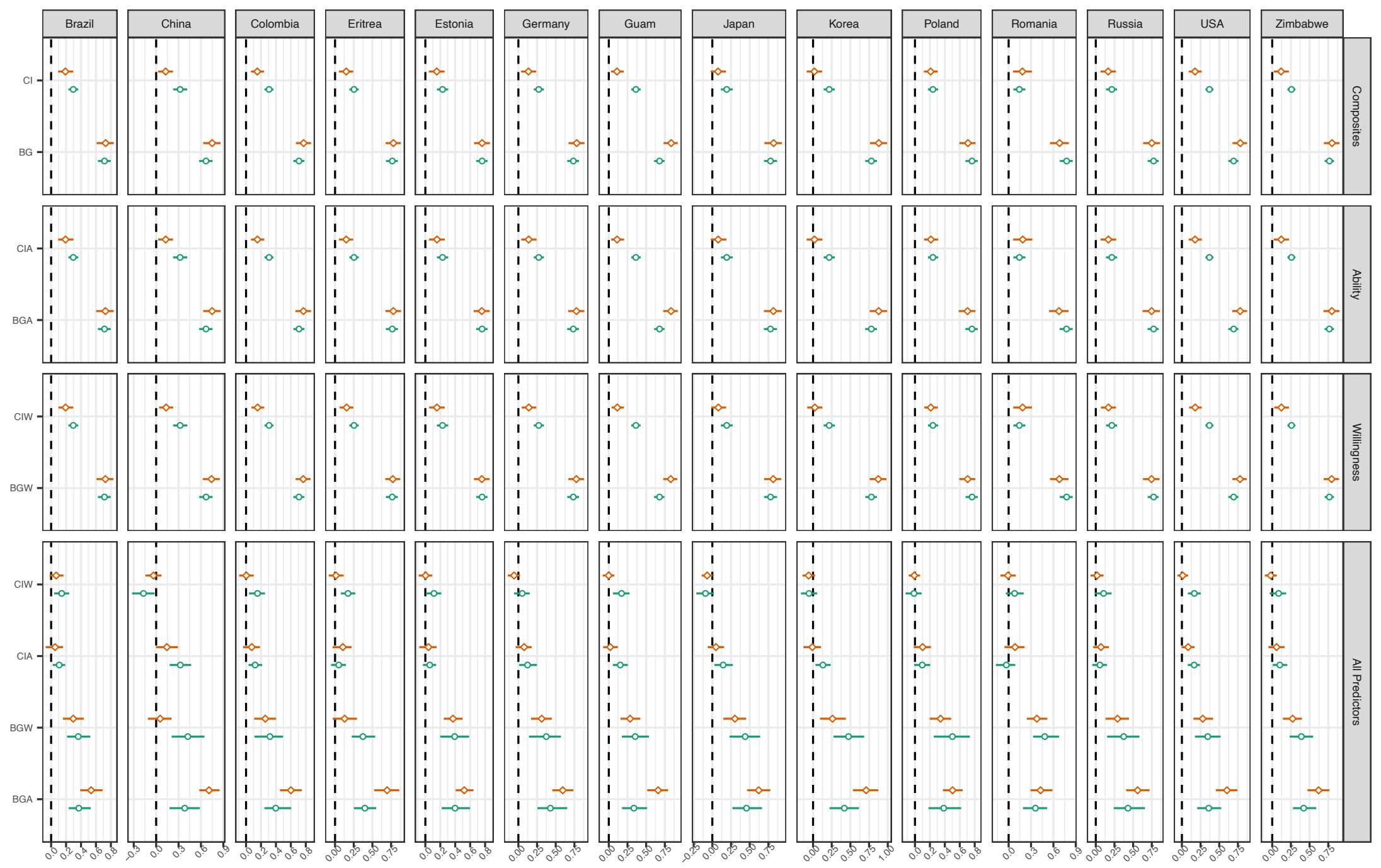

Ridge Coefficient

\& Men's Status \& Women's Status

Figure 4. Ridge regression results across 2000 bootstrapped replicates, showing the mean and 95\% CI coefficient estimates across different combinations of predictors of men's and women's status across nations. 


\section{Random Forests}

Random forest models are a type of supervised machine learning method that build accurate predictive classification models (Brieman, 2001). Provided a dataset, a defined set of predictors, and a criterion, random forests build a "forest" of classification or regression (depending on whether the criterion is continuous or categorical) decision trees based on different bootstrapped samples of the data (for a complete overview, see Strobl et al., 2009b). In each tree, the predictors of interest are recursively partitioned, with binary splits, on some arbitrary value of a random predictor (e.g., above and below .5 standard deviations above the mean for BGA). After each split, the cases in the resulting group are assigned the average value of the criterion (e.g., status rating) as the predicted value for that node in the decision tree. These resulting groups are then split again on another random-selected predictor and assigned new predicted values based on the average of the criterion for cases in each split group. This process is repeated iteratively until all cases are classified with $100 \%$ accuracy or some other stopping criteria is met. The resulting fitted values across all the decision trees that make up the random forest ensemble are more accurate than any individual tree in the forest. In random forest models, the available predictors to select from in each tree is typically also randomized so only a subset of predictors is available to each tree (and its nodes), which helps to diversify the forest and decrease error in the aggregate.

The relative importance of predictors can be assessed in random forests by randomizing values on one predictor variable at a time across all decision trees. The loss of prediction accuracy across all trees indicates the extent to which the predictor is necessary for accurate prediction. If assigning random values to a predictor does not decrease the prediction accuracy, it is likely not that important of a predictor. The resulting permutation variable importance indices are relative values that cannot be interpreted as absolute values. Simulations of permutation importance show that it overestimates the importance of correlated predictors (Strobl et al., 2007), so it is recommended that a conditional variable importance measure developed by Strobl et al. (2008) is used when predictors are correlated. These procedures have been used to examine the relative importance of highly correlated predictors in several psychological studies (e.g., Götz et al., 2020; IJzerman et al., 2018; Matsuki et al., 2016).

We conducted random forest regression to predict the impact of different personal characteristics on men's and women's status in each nation from the perceptions of those characteristics as being indicative of men's and women's ability to inflict costs on others (CIA), willingness to inflict costs on others (CIW), ability to generate benefits for others (BGA), and willingness to generate benefits for others (BGW). We used the $\mathrm{R}$ package party to estimate the random forests with the cforest function and assess conditional permutation variable importance with the varimp function (Strobl et al., 2009a). The cforest function employs conditional inference trees, which differ somewhat from traditional random forest algorithms in that they employ significance tests to determine split points and variables. We set each conditional inference forest to grow 2000 trees and created three different forests that sampled only either two, three, or four of our predictors in each tree - this allows us to confirm that the relative ranking of variable importance is consistent across diverse trees, as is recommended (Strobl et al., 2009a).

Figure 5 shows the relative importance of our predictors for men's and women's status in each nation, when different numbers of randomly selected variables were used to build trees (i.e., mtry). The relative rankings of variable importance are stable across different levels of mtry, as well as under different random seeds, suggesting that the results are reliable. The results suggest that both benefit generation variables are consistently more important predictors of men's and women's status across nations than are the cost infliction variables, mirroring our original analyses and conclusions and converging with results found with dominance analysis, commonality analysis, and ridge regression. 

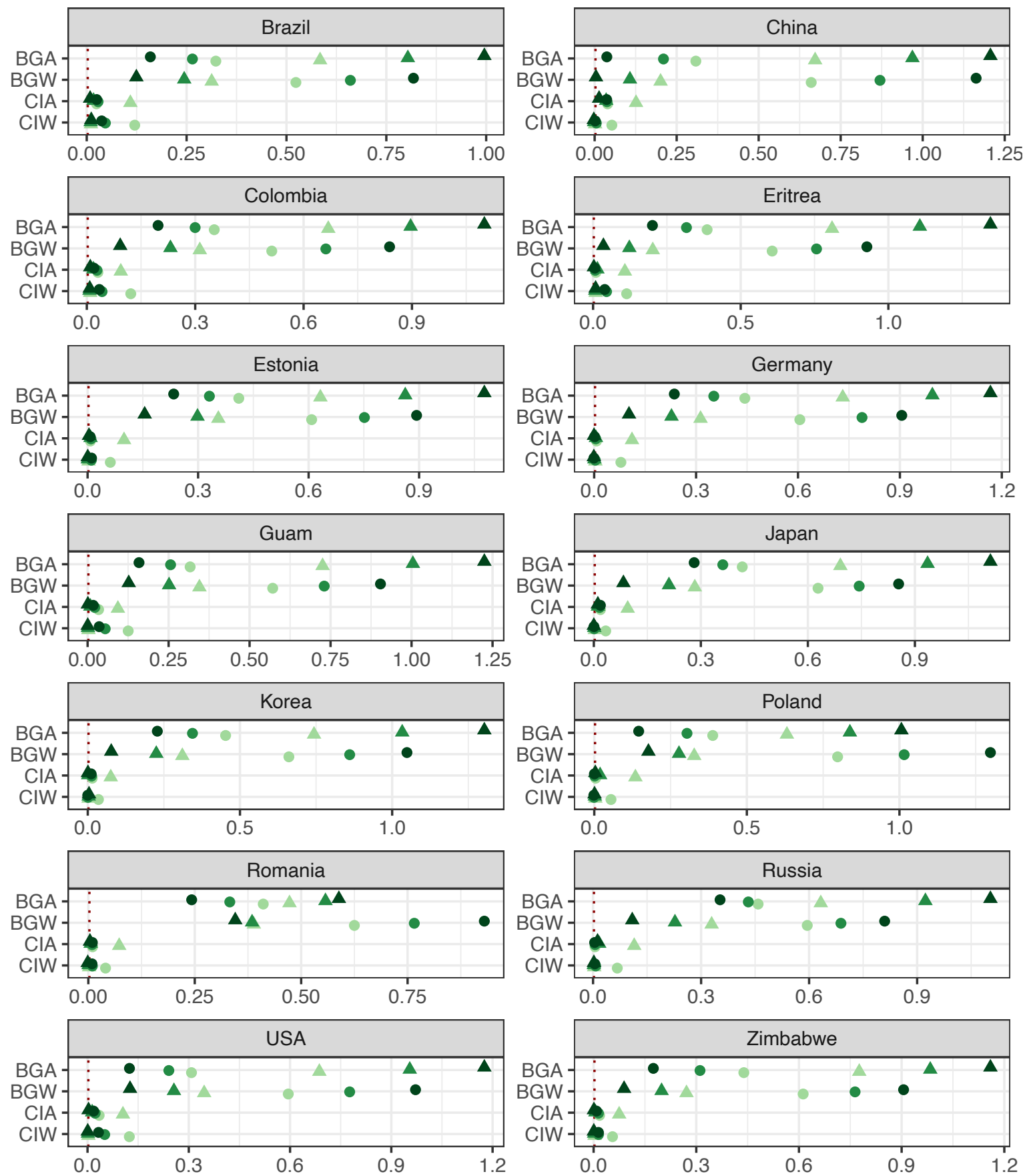

Conditional Variable Importance

- Men's Status $\Delta$ Women's Status

mtry: $2 \bullet 3 \bullet 4$

Figure 5. Conditional variable importance estimates for predictors of men's and women's status in each nation under different levels of mtry (i.e., the number of variables available to be randomly selected in each regression tree). The red line indicates the threshold at which a variable's importance is distinguishable from random noise. 


\section{General Conclusion}

This extended reply to CTH's comment on our original paper (Durkee et al., 2020) presented further examination of the relative importance of cost infliction and benefit generation in human status attainment (see also, Durkee et al., 2021). We conducted re-analyses of our cross-national status data - which each addressed the collinearity among our predictions in different ways - and found continued support for our original conclusion that benefit generation is a more crucial foundation of status allocation in human groups than cost infliction. We also proposed a distinction between pure cost infliction (PCI) and benefit generation via cost infliction (BGCI) to explain why "dominance," as it has been operationalized in extant empirical studies, might be associated with status in human groups. We hope that our original data on the inferential foundations of status allocations and this extended discussion of the empirical and conceptual issues with "dominance" can help improve future investigations of human hierarchies and their psychological underpinnings. 


\section{References}

Anderson, C., Sharps, D. L., Soto, C. J., \& John, O. P. (2020). People with disagreeable personalities (selfish, combative, and manipulative) do not have an advantage in pursuing power at work. Proceedings of the National Academy of Sciences, 117(37), 22780-22786.

Azen, R., \& Budescu, D. V. (2003). The dominance analysis approach for comparing predictors in multiple regression. Psychological methods, 8(2), 129.

Breiman, L. (2001). Random forests. Machine learning, 45(1), 5-32.

Boehm, C. (1993). Egalitarian society and reverse Dominance hierarchy. Current Anthropology, 34, 227-254. doi:10.1086/204166

Budescu, D. V. (1993). Dominance analysis: a new approach to the problem of relative importance of predictors in multiple regression. Psychological bulletin, 114(3), 542.

Buss, D. M., Durkee, P. K., Shackelford, T. K., Bowdle, B. F., Schmitt, D. P., Brase, G. L., ... \& Trofimova, I. (2020). Human status criteria: Sex differences and similarities across 14 nations. Journal of Personality and Social Psychology.

Cheng, J. T. (2020). Dominance, prestige, and the role of leveling in human social hierarchy and equality. Current opinion in psychology, 33, 238-244.

Cheng, J. T., Tracy, J. L., Foulsham, T., Kingstone, A., \& Henrich, J. (2013). Two ways to the top: evidence that dominance and prestige are distinct yet viable avenues to social rank and influence. Journal of personality and social psychology, 104(1), 103.

Cheng, J. T., Tracy, J. L., \& Henrich, J. (2010). Pride, personality, and the evolutionary foundations of human social status. Evolution and Human Behavior, 31(5), 334-347.

Cheng, J. T., Tracy, J. L., \& Henrich, J. (2021 $\left.{ }^{\mathrm{a}}\right)$. Dominance is necessary to explain human status hierarchies. Proceedings of the National Academy of Sciences.

Cheng, J. T., Tracy, J., \& Henrich, J. (2021 ${ }^{\mathrm{b}}$, March 26). Dominance is necessary to explain human status hierarchies - Extended online version and supplemental. https://doi.org/10.31234/osf.io/w28nt

Durkee, P. K., Lukaszewski, A. W., \& Buss, D. M. (2020). Psychological foundations of human status allocation. Proceedings of the National Academy of Sciences, 117(35), 21235-21241.

Durkee, P. K., Lukaszewski, A. W., \& Buss, D. M. (2021). Reply to Cheng et al.: Benefit generation dominates cost infliction in explaining cross-national status allocations. Proceedings of the National Academy of Sciences.

Garfield, Z. H., \& Hagen, E. H. (2020). Investigating evolutionary models of leadership among recently settled Ethiopian hunter-gatherers. The Leadership Quarterly, 31(2), 101290.

Garfield, Z. H., Syme, K. L., \& Hagen, E. H. (2020). Universal and variable leadership dimensions across human societies. Evolution and Human Behavior, 41(5), 397-414.

Gintis, H., Van Schaik, C., Boehm, C., Chapais, B., Flack, J. C., Pagel, M., ... \& Boehm, C. (2015). Zoon politikon: The evolutionary origins of human political systems. Current Anthropology, 56(3), 340-341.

Golub, G. H., Heath, M., \& Wahba, G. (1979). Generalized cross-validation as a method for choosing a good ridge parameter. Technometrics, 21(2), 215-223.

Götz, F. M., Stieger, S., Gosling, S. D., Potter, J., \& Rentfrow, P. J. (2020). Physical topography is associated with human personality. Nature human behaviour, 4(11), 1135-1144.

Hoerl, A. E., \& Kennard, R. W. (1970). Ridge regression: Biased estimation for nonorthogonal problems. Technometrics, 12(1), 55-67.

IJzerman, H., Lindenberg, S., Dalğar, İ., Weissgerber, S. S., Vergara, R. C., Cairo, A. H., ... \& Mccarthy, R. (2018). The Human Penguin Project: Climate, social integration, and core body temperature. Collabra: Psychology, 4(1).

Jernstedt, G. C. (1980). Commonality analysis: Partitioning variance for multivariate prediction. Educational and Psychological measurement, 40(3), 739-743.

Jiménez, Á. V., Flitton, A., \& Mesoudi, A. (2021). When do people prefer dominant over prestigious political leaders?. Evolutionary Human Sciences, 3.

Henrich, J., \& Gil-White, F. J. (2001). The evolution of prestige: Freely conferred deference as a mechanism for enhancing the benefits of cultural transmission. Evolution and human behavior, 22(3), 165-196.

Hess, N., Helfrecht, C., Hagen, E., Sell, A., \& Hewlett, B. (2010). Interpersonal aggression among Aka huntergatherers of the Central African Republic. Human Nature, 21(3), 330-354.

Kakkar, H., \& Sivanathan, N. (2017). When the appeal of a dominant leader is greater than a prestige leader. Proceedings of the National Academy of Sciences, 114(26), 6734-6739. 
Kakkar, H., Sivanathan, N., \& Gobel, M. S. (2020). Fall from grace: The role of dominance and prestige in the punishment of high-status actors. Academy of Management Journal, 63(2), 530-553.

Ketterman, A. B., \& Maner, J. K. (2021). Complaisant or coercive? The role of dominance and prestige in social influence. Personality and Individual Differences, 177, 110814.

Kraha, A., Turner, H., Nimon, K., Zientek, L., \& Henson, R. (2012). Tools to support interpreting multiple regression in the face of multicollinearity. Frontiers in psychology, 3, 44.

Krzywinski, M., Altman, N. (2017) Classification and regression trees. Nature Methods, 14, 757-75.

Lukaszewski, A. W. (2013). Testing an adaptationist theory of trait covariation: Relative bargaining power as a common calibrator of an interpersonal syndrome. European Journal of Personality, 27(4), 328-345.

Lukaszewski, A. W., Simmons, Z. L., Anderson, C., \& Roney, J. R. (2016). The role of physical formidability in human social status allocation. Journal of Personality and Social Psychology, 110(3), 385.

Maner, J. K., \& Mead, N. L. (2010). The essential tension between leadership and power: when leaders sacrifice group goals for the sake of self-interest. Journal of Personality and Social Psychology, 99(3), 482.

Matsuki, K., Kuperman, V., \& Van Dyke, J. A. (2016). The Random Forests statistical technique: An examination of its value for the study of reading. Scientific Studies of Reading, 20(1), 20-33.

Navarrete, C. B., \& Soares, F. C. (2020). dominanceanalysis: Dominance Analysis. R package version 2.0.0. https://CRAN.R-project.org/package=dominanceanalysis

Nimon, K., Lewis, M., Kane, R. \& Haynes, R. M. (2008) An R package to compute commonality coefficients in the multiple regression case: An introduction to the package and a practical example. Behavior Research Methods, 40, 457-466.

Polman, E., Pettit, N. C., \& Wiesenfeld, B. M. (2013). Effects of wrongdoer status on moral licensing. Journal of Experimental Social Psychology, 49(4), 614-623.

Ray-Mukherjee, J., Nimon, K., Mukherjee, S., Morris, D. W., Slotow, R., \& Hamer, M. (2014). Using commonality analysis in multiple regressions: a tool to decompose regression effects in the face of multicollinearity. Methods in Ecology and Evolution, 5(4), 320-328.

Redhead, D. J., Cheng, J. T., Driver, C., Foulsham, T., \& O'Gorman, R. (2019). On the dynamics of social hierarchy: A longitudinal investigation of the rise and fall of prestige, dominance, and social rank in naturalistic task groups. Evolution and Human Behavior, 40(2), 222-234.

Ripley, B., Venables, B., Bates, D. M., Hornik, K., Gebhardt, A., Firth, D., \& Ripley, M. B. (2013). Package 'MASS'. Cran R, 538, 113-120.

Sell, A., Eisner, M., \& Ribeaud, D. (2016). Bargaining power and adolescent aggression: The role of fighting ability, coalitional strength, and mate value. Evolution and Human Behavior, 37(2), 105-116.

Sell, A., Tooby, J., \& Cosmides, L. (2009). Formidability and the logic of human anger. Proceedings of the National Academy of Sciences, 106(35), 15073-15078.

Singh, M. (2018). The cultural evolution of shamanism. Behavioral and Brain Sciences, 41.

Strobl, C., Boulesteix, A. L., Kneib, T., Augustin, T., \& Zeileis, A. (2008). Conditional variable importance for random forests. BMC bioinformatics, $9(1), 1-11$.

Strobl, C., Boulesteix, A. L., Zeileis, A., \& Hothorn, T. (2007). Bias in random forest variable importance measures: Illustrations, sources and a solution. BMC bioinformatics, 8(1), 1-21.

Strobl, C., Hothorn, T., \& Zeileis, A. (2009a). Party on! A new, conditional variable importance measure available in the party package. The R Journal, (2), 14-17.

Strobl, C., Malley, J., \& Tutz, G. (2009b). An introduction to recursive partitioning: rationale, application, and characteristics of classification and regression trees, bagging, and random forests. Psychological methods, 14(4), 323.

Ullah, M. I., Aslam, M., \& Altaf, S. (2018). lmridge: A Comprehensive R Package for Ridge Regression. $R$ J., $10(2), 326$.

Vanhove, J. (2021). Collinearity isn't a disease that needs curing. Meta-Psychology, 5.

von Rueden, C., Gurven, M., \& Kaplan, H. (2008). The multiple dimensions of male social status in an Amazonian society. Evolution and Human Behavior, 29(6), 402-415.

von Rueden, C., Gurven, M., Kaplan, H., \& Stieglitz, J. (2014). Leadership in an egalitarian society. Human Nature, 25(4), 538-566.

von Rueden, C. R., Redhead, D., O'Gorman, R., Kaplan, H., \& Gurven, M. (2019). The dynamics of men's cooperation and social status in a small-scale society. Proceedings of the Royal Society B, 286(1908), 20191367.

Wrangham, R. W. (2021). Targeted conspiratorial killing, human self-domestication and the evolution of groupishness. Evolutionary Human Sciences, 3. 
*Address correspondence to Patrick Durkee (pdurkee@utexas.edu) 